\title{
Spectral-Fluctuations Test of the Quark-Model Baryon Spectrum
}

\author{
C. Fernández-Ramírez ${ }^{*}$ and A. Relaño ${ }^{\dagger}$ \\ Instituto de Estructura de la Materia, CSIC, Serrano 123, E-28006 Madrid, Spain
}

(Received 27 November 2006; published 8 February 2007)

\begin{abstract}
We study the low-lying baryon spectrum (up to $2.2 \mathrm{GeV}$ ) provided by experiments and different quark models using statistical tools which allow us to postulate the existence of missing levels in spectra. We confirm that the experimental spectrum is compatible with random matrix theory, the paradigmatic model of quantum chaos, and we find that the quark models are more similar to a Poisson distribution, which is not compatible with what should be expected in a correlated spectrum. From our analysis it stems that the spectral fluctuation properties of quark-model spectra are incompatible with experimental data. This result can be used to enlighten the problem of missing resonances.
\end{abstract}

DOI: 10.1103/PhysRevLett.98.062001

PACS numbers: $14.20 .-\mathrm{c}, 05.45 . \mathrm{Mt}, 12.39 . \mathrm{Ki}$

Since the discovery of the first excited state of the nucleon, the $\Delta(1232)$ [1], baryons have played a central role in the study of the strong interaction. After this discovery, baryons proliferated and their mass spectrum attracted a lot of attention. In the 1960s and 1970s, nonrelativistic quark models were worked out [2] and the quark-model framework was established, contributing strongly to the development of quantum chromodynamics (QCD). Later on, quark models evolved into relativistic versions [3-5].

It is well known that the number of baryons predicted by quark models [2-5] is substantially larger than what is observed in meson scattering and production experiments [6]. This fact raises the problem of missing resonances, which has opened the door to a huge experimental effort in recent years to observe and identify these missing states [7]. These experiments have to achieve high precision due to the important background (which can veil resonances) and the overlap of baryons, as well as the need to survey different meson production channels and observables. The procedure to assess the existence of these elusive baryons consists on analyses of partial waves [8] and polarization observables [9] of the reactions comparing experimental data from different sources to what is obtained after including or removing the hypothetical resonance. If data are better reproduced, the existence of a resonance is possible but sometimes debatable (the pentaquark search is a very good example of how difficult these studies are [10]). The Particle Data Group (PDG) rates the possible existence of the resonances based on the quantity and quality of experimental data. Only after several independent experiments and analyses, a baryon is awarded a well-established status, rated with three or four stars.

In the last years, lattice QCD (LQCD) [11] and effective QCD-inspired models (EQCDiM) [12] have started to provide insight on the nonperturbative energy regime of quantum chromodynamics (QCD) where the low-lying baryons live, but they are still far away from providing a complete analysis. Hence, we have to resort to tractable models, such as quark models, to study the baryon spectrum. For this reason, quark models are expected to retain in forthcoming years the importance they had in the study of the static properties and internal structure of baryons.

It is well established that baryons are aggregates of partons. Consequently, the mass spectrum of low-lying baryons is an energy spectrum of a standard many-body quantum system, like an atomic nucleus, and it consists on all the possible bound and excited states which stem from an interacting many-body quantum system. Since Wigner discovered that the statistical properties of complex nuclear spectra are well described by random matrix theory (RMT) [13], statistical methods have become a powerful tool to study the energy spectra of quantum systems. The most striking result in this field is that the statistical properties of the energy-level fluctuations are universal and determine if a system is chaotic or integrable. Integrable systems display a noncorrelated sequence of levels, which follows the Poisson distribution [14], whereas chaotic systems are characterized by a correlation structure described by RMT [15]. This kind of analysis has been already applied to the hadron mass spectrum by Pascalutsa [16] obtaining a chaoticlike behavior.

In this Letter, we apply the spectral statistical techniques to the problem of missing resonances. Recent works by Bohigas and Pato [17] prove that if we randomly remove some energy levels from a correlated spectrum, it partially loses correlations and becomes closer to a Poisson distribution regardless of its actual correlation structure. Following this result, we can obtain hints on the existence of missing resonances comparing the spectral correlations of the baryon spectra supplied by experiments and quark models. We have analyzed the experimental spectrum (Breit-Wigner masses) provided by the PDG (set EXP in what follows) [6] and the spectra given by the relativistic quark models by Capstick and Isgur (set CI) [3] and by Löring et al. [5] (sets L1 and L2 which correspond, respectively, to models $\mathcal{A}$ and $\mathcal{B}$ in [5]). We have considered all the resonance states in these spectra up to $2.2 \mathrm{GeV}$. In principle, if the experimental spectrum is not complete due 


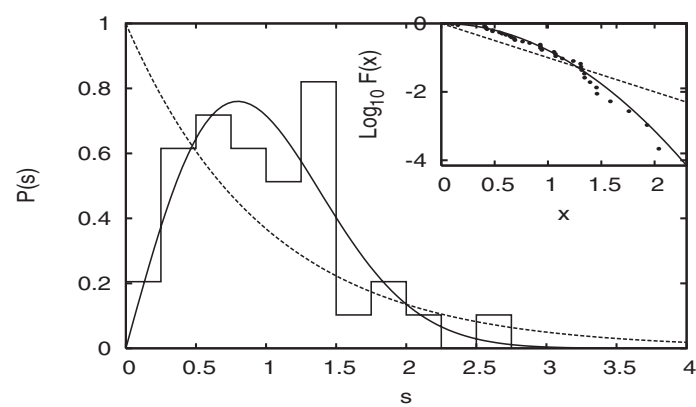

(a) Experimental values from PDG (set EXP).

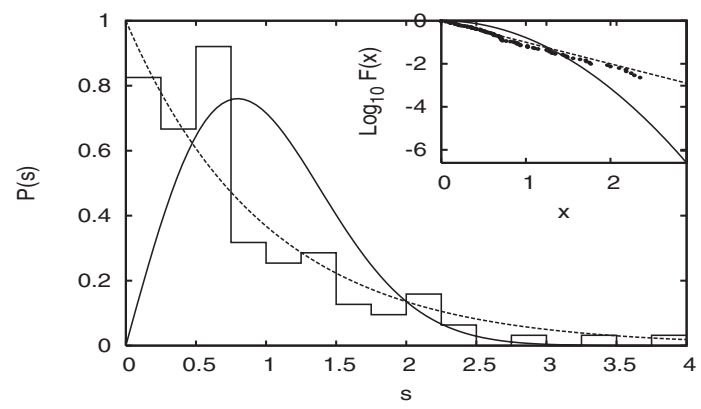

(b) Model by Capstick and Isgur (set CI).

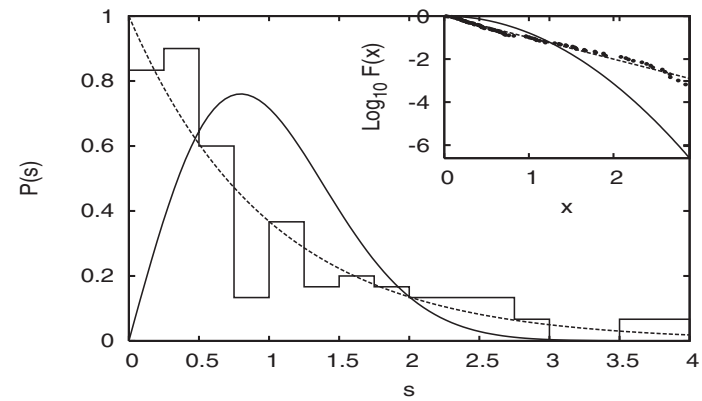

(c) Model by Löring et al. (set L1).

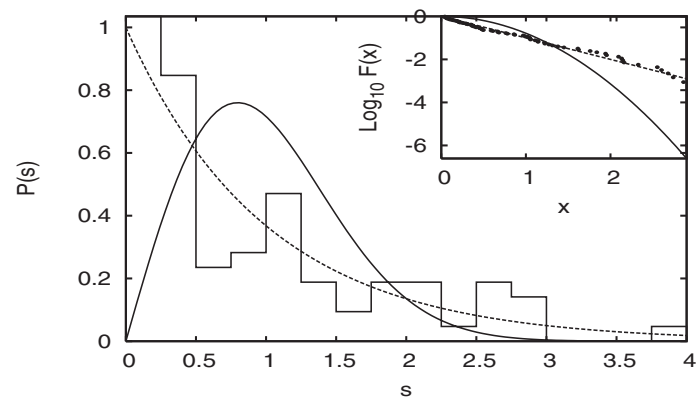

(d) Model by Löring et al. (set L2).

FIG. 1. NNSD for the experimental spectrum provided by the PDG data, the model by Capstick and Isgur, and two parametrizations of the model by Löring et al. The histogram represents the spacings; the solid line, the Wigner surmise; and the dashed line, the Poisson distribution. The inset shows the function $F(x)$ in logarithmic scale. to the absence of some baryons which are not observed but are predicted by quark models, and if we assume that missing resonances are randomly distributed [18], the experimental spectrum should be less correlated than the theoretical ones.

Prior to any statistical analysis we have to accomplish two preliminary tasks. First of all, it is necessary to identify the different symmetries involved in the spectra. If a sequence of levels involves more than one symmetry, its spectral statistics are deflected towards a Poisson distribution (see [13,20] for generic reviews and [19] for a recent work where the effects of both mixing symmetries and missing levels in the same sequence are surveyed). Hence, it is necessary to extract from the full spectrum sequences of levels involving the same symmetries (quantum numbers) to proceed with the spectral analysis.

In the spectra considered in this Letter, we can identify the following symmetries associated to the baryons: spin, isospin, parity, and strangeness. Strangeness can be dropped due to the assumption of flavor $S U(3)$ invariance. Therefore, for every statistical analysis, we split all the spectra in sequences where all the levels present the same values of spin, isospin, and parity. For reasons stated below we only account for sequences with three or more levels.

The second preliminary task is the unfolding procedure. In any energy-level spectrum, we can split the level density $\rho(E)$ into a smooth part $\bar{\rho}(E)$ and a fluctuating part $\tilde{\rho}(E)$, with $\rho(E)=\bar{\rho}(E)+\tilde{\rho}(E)$. The unfolding procedure allows one to extract the fluctuating part from the level density, removing the smooth component of the spectrum. There are several ways to unfold a spectrum and we choose the simplest one. First, we compute the distance between two consecutive levels, $S_{i}=E_{i+1}-E_{i}$, and then we rescale $S_{i}$ using its average value $s_{i}=S_{i} /\langle S\rangle$ [21]. The resulting quantities are called nearest neighbor spacings (NNS). This procedure undergoes some problems, especially in the long-range correlation analysis [22], but it is suitable for the kind of analysis of our concern in this Letter.

From the NNS we obtain one of the most relevant quantities in spectral statistical analysis: the nearest neighbor spacing distribution (NNSD). The NNSD follows the Poisson distribution $P(s)=\exp (-s)$ if the spectrum is integrable (noncorrelated) [14], but it follows the Wigner surmise $P(s)=\frac{\pi s}{2} \exp \left(-\frac{\pi s^{2}}{4}\right)$, which stems from RMT, for a chaotic (correlated) spectrum [15]. For our purpose here, it is enough to consider that the less (more) correlated the sequence of levels is, the closer to the Poisson (Wigner) distribution the NNSD is.

In order to obtain a significative result we have calculated the NNSD, $P(s)$, for each one of the four different spectra we survey: EXP, CI, L1, and L2. We account for all the sequences $\left\{s_{i}\right\}_{X}$, where $X$ stands for the quantum numbers which identify each sequence [23]. Set EXP has 70 energy levels distributed in 15 sequences; set CI, 145 levels 
and 19 sequences; set L1, 142 levels and 21 sequences; and set L2, 104 levels and 19 sequences.

We also have evaluated the function

$$
F(x)=1-\int_{0}^{x} d s P(s)
$$

which is related to the accumulated NNSD and allows a better study of the tail of the distribution.

Results are depicted in Fig. 1. The experimental spectrum is closer to the Wigner surmise than to the Poisson distribution (in good agreement with what Pascalutsa obtains in [16]). On the other hand, the three theoretical spectra are quite close to the Poisson distribution, so that they are less correlated than the experimental spectrum. These results are opposite to what is expected from an experimental spectrum spoiled by missing resonances. If the experimental spectrum is not complete due to missing states, it has to be closer to the Poisson distribution than the theoretical ones.

However, there is one subtle objection to our analysis regarding the unfolding procedure which has to be considered in detail. As we have pointed before, the unfolding has to be performed independently for each $\left\{s_{i}\right\}_{X}$ sequence. This entails that, at least in some cases, we have worked with very short sequences of levels. In such cases, the unfolding procedure could give rise to misleading results if it makes the spacings $s_{i}$ spuriously closer to $s_{i} \simeq 1$, shifting the NNSD far away from the Poisson distribution and bringing it to the Wigner surmise (roughly centered at $s=1$ ). As an extreme example, let us see what happens to a sequence of two levels after the unfolding. In this case, we only have $S_{1}=E_{2}-E_{1}$, and, therefore, $s_{1}=$ $S_{1} /\langle S\rangle=1$. This is the reason why we have included only sequences with three or more levels in our analysis. Moreover, since the experimental sequences are shorter than the theoretical ones, they can be more affected by this problem, spoiling the direct comparison we have made above. Therefore, to reach a final conclusion it is mandatory to perform an improved analysis.

To elucidate whether the unfolding procedure can give rise to misleading results, we have accomplished a more sophisticated analysis of the NNSD. Since the unfolding could yield different effects on experimental and theoretical spectra, we have avoided a direct comparison among them. Instead, we have built four Poisson-like and four RMT-like reference spectra, each of them optimized to study each one of the four sets of data (EXP, CI, L1, and L2). We have proceeded in the following way for every set. For each sequence $\left\{s_{i}\right\}_{X}$ of a given spectrum we have built two equivalent reference sequences $\left\{s_{i}^{\prime}\right\}_{X}$ with the same length, one RMT-like and another Poisson-like. In doing so, we start from one long RMT-like spectrum and one long Poisson-like spectrum. We divide each spectrum in as many subsequences as sets with the same quantum numbers the spectrum under consideration has, each one with its appropriate length. Next, we unfold each reference sequence independently and calculate the NNSD for each set. Finally, we compare each spectrum obtained directly from the experimental data (EXP) and the quark models (CI, L1, and L2) to the two ad hoc built-up RMT-like and Poisson-like reference spectra. In this way, each reference spectra is distorted by the unfolding in the same way as sets EXP, CI, L1, and L2 are. Consequently, we can verify whether their correlations are true or spurious due to the unfolding.

To obtain significative results, we have performed the Kolmogorov-Smirnov distribution test [24] in each case. As null hypothesis we have chosen that the studied NNSD coincides with the ad hoc built-up reference Poisson-like or RMT-like distributions, against the hypothesis that both distributions are different. In Table I we summarize our results. We observe that all the spectra emerging from the three theoretical models are incompatible with RMT, whereas the experimental one seems to be closer to the Wigner surmise than to the Poisson distribution. Set L2 provides a result that seems to be incompatible with both Poisson and RMT behaviors. From panel (d) in Fig. 1 it stems that the probability to observe spacings closer to zero are higher than for a Poisson distribution. Hence, the deviation from a Wigner surmise is even larger than from a Poisson distribution.

These results, together with those shown in Fig. 1, point out that the experimental spectrum is more correlated than the three theoretical ones. Following the work of Bohigas and Pato [17], we can say that this is incompatible with the usual statement that there are missing resonances in the experimental spectrum which are included in the theoretical models. Moreover, the Hamiltonians used in such models do not describe the statistical properties of the experimental spectrum; they rather correspond to an integrable system while the experimental spectrum is close to a chaotic system. Hence, quark models, as they are presently built, may not be suitable to reproduce the low-lying baryon spectrum, and, therefore, to predict the existence of missing resonances.

On the other hand, it is important to notice that quark models assume triality (three-quark states), while QCD allows non-three-quark states such as pentaquarks and hybrid baryons (excited glue). Therefore, according to QCD degrees of freedom, the spectrum which is obtained within quark models cannot be complete. Consequently, the NNSD of any quark model (whatever the interacting

TABLE I. Probability to obtain, under the null hypothesis, a value of the Kolmogorov-Smirnov test statistic as extreme as that observed.

\begin{tabular}{lcccc}
\hline \hline Spectrum & EXP & CI & L1 & L2 \\
\hline Poisson & 0.25 & 0.44 & 0.37 & 0.10 \\
RMT & 0.56 & $4.5 \times 10^{-5}$ & $1.3 \times 10^{-4}$ & $6.5 \times 10^{-3}$ \\
\hline \hline
\end{tabular}


Hamiltonian is) may be deflected to a Poisson distribution due to its inherent incompleteness. The importance and quantification of such an effect depends on the amount of missing non-three-quark states present in the actual spectrum and remains as an open question. However, we cannot blame the non-Wigner character obtained for the quark models studied in this Letter on this effect: the loss of correlation cannot be due to missing non-three-quark states because theoretical models predict more levels than what is observed.

Regardless we obtain that present quark models are not able to predict missing resonances in the experimental spectrum, our results are compatible with the existence of some missing states. In fact, the shape of the NNSD and the Kolmogorov-Smirnov test for a RMT-like spectrum with a $20 \%$ of random missing levels are alike to those of set EXP.

The analysis presented in this Letter should be extended to baryon spectra provided by LQCD and EQCDiM as soon as complete calculations become available. In this way we can use the universal properties of fluctuations to examine the results given by these models or to use the spectra provided by LQCD and EQCDiM to test the universality of fluctuations.

The authors thank Dr. T. van Cauteren, Dr. F. J. LlanesEstrada, Dr. R. A. Molina, Professor E. Moya de Guerra, Dr. J. Retamosa, and Dr. J. M. Udías for valuable comments. A. R. is supported by the Spanish program "Juan de la Cierva." This work has been partly supported under contracts of Ministerio de Educación y Ciencia (Spain) No. FTN2003-08337-C04-04 and No. FIS2005-00640.

*Corresponding author (baryons).

Electronic address: cesar@nuc2.fis.ucm.es

Corresponding author (quantum chaos and statistical tools)

Electronic address: armando@iem.cfmac.csic.es

[1] H. L. Anderson, E. Fermi, E. A. Long, and D. E. Nagle, Phys. Rev. 85, 936 (1952).

[2] G. Zweig, CERN Report No. TH-401, 1964; CERN Report No. TH-412, 1964; O. W. Greenberg and M. Resnikoff, Phys. Rev. 163, 1844 (1967); R. Horgan and R. H. Dalitz, Nucl. Phys. B66, 135 (1973); N. Isgur and G. Karl, Phys. Rev. D 18, 4187 (1978); Phys. Rev. D 20, 1191 (1979); R. Koniuk and N. Isgur, Phys. Rev. D 21, 1868 (1980); A. J. Hey and R. L. Kelly, Phys. Rep. 96, 71 (1983).

[3] S. Capstick and N. Isgur, Phys. Rev. D 34, 2809 (1986).

[4] S. Capstick and W. Roberts, Prog. Part. Nucl. Phys. 45, S241 (2000).

[5] U. Löring, K. Kretzschmar, B.Ch. Metsch, and H. R. Petry, Eur. Phys. J. A 10, 309 (2001); U. Löring, B. Ch. Metsch, and H. R. Petry, Eur. Phys. J. A 10, 395 (2001); Eur. Phys. J. A 10, 447 (2001); U. Löring, Ph.D. dissertation, Universität Bonn, Germany, 2001.

[6] W.-M. Yao et al., J. Phys. G 33, 1 (2006).
[7] M. Q. Tran et al., Phys. Lett. B 445, 20 (1998); E. Anciant et al., Phys. Rev. Lett. 85, 4682 (2000); K. Lukashin et al., Phys. Rev. C 63, 065205 (2001); 64, 059901(E) (2001); B. Krusche and S. Schadmand, Prog. Part. Nucl. Phys. 51, 399 (2003); J. W. C. McNabb et al., Phys. Rev. C 69, 042201(R) (2004); V. Crede et al., Phys. Rev. Lett. 94, 012004 (2005); R. Bradford et al., Phys. Rev. C 73, 035202 (2006).

[8] T. P. Vrana, S. A. Dytman, and T.-S.H. Lee, Phys. Rep. 328, 181 (2000); R. A. Arndt, W. J. Briscoe, I. I. Strakovsky, and R.L. Workman, Phys. Rev. C 66, 055213 (2002); http://gwdac.phys.gwu.edu/.

[9] D. Dutta, H. Gao, and T.-S.H. Lee, Phys. Rev. C 65, 044619 (2002).

[10] T. Nakano et al., Phys. Rev. Lett. 91, 012002 (2003); J. M. Link et al., Phys. Lett. B 622, 229 (2005); M. Battaglieri et al., Phys. Rev. Lett. 96, 042001 (2006); R. De Vita et al., Phys. Rev. D 74, 032001 (2006); B. McKinnon et al., Phys. Rev. Lett. 96, 212001 (2006); J. M. Link et al., Phys. Lett. B 639, 604 (2006); M. Ripani et al., Phys. Rev. Lett. 91, 022002 (2003).

[11] C. Bernard, T. Burch, K. Orginos, D. Toussaint, T. A. DeGrand, C. DeTar, S. Datta, S. Gottlieb, U. M. Heller, and R. Sugar, Phys. Rev. D 64, 054506 (2001).

[12] P. R. Page, E.S. Swanson, and A. P. Szczepaniak, Phys. Rev. D 59, 034016 (1999); F. J. Llanes-Estrada and S. R. Cotanch, Phys. Rev. Lett. 84, 1102 (2000); Phys. Lett. B 504, 15 (2001); Nucl. Phys. A697, 303 (2002).

[13] C.E. Porter, Statistical Theories of Spectra: Fluctuations (Academic Press, New York, 1965).

[14] M. V. Berry and M. Tabor, Proc. R. Soc. A 356, 375 (1977).

[15] O. Bohigas, M.J. Giannoni, and C. Schmit, Phys. Rev. Lett. 52, 1 (1984).

[16] V. Pascalutsa, Eur. Phys. J. A 16, 149 (2003).

[17] O. Bohigas and M. P. Pato, Phys. Lett. B 595, 171 (2004); Phys. Rev. E 74, 036212 (2006).

[18] In Ref. [19] the effects of removing levels in a nonstrictly random way have been studied. It is shown that if the probability to find a level depends linearly on the energy, the correlations are roughly lost as predicted by Bohigas and Pato [17].

[19] R. A. Molina, J. Retamosa, L. Muñoz, A. Relaño, and E. Faleiro, Phys. Lett. B 644, 25 (2007).

[20] T. Guhr, A. Müller-Groeling, and H. A. Weidenmüller, Phys. Rep. 299, 189 (1998); M.L. Mehta, Random Matrices (Academic Press, New York, 1991).

[21] This procedure assumes an energy independent behavior of the smooth part of the density $\bar{\rho}(E)=1 /\langle S\rangle$. The validity of this hypothesis was confirmed by Pascalutsa in [16].

[22] J.M. G. Gómez, R. A. Molina, A. Relaño, and J. Retamosa, Phys. Rev. E 66, 036209 (2002).

[23] In doing so, we assume that every sequence $\left\{s_{i}\right\}_{X}$ coming from the same spectra has the same NNSD. This is a realistic assumption, since we want to calculate the statistical properties of each spectrum as a whole.

[24] Numerical Algorithms Group Ltd., Wilkinson House, Oxford, Jordan Hill Road, OX2-8DR, U.K., http:// www.nag.co.uk/ 